\title{
Nonequilibrium dynamics of urn models
}

\author{
C Godrèchet眜 and J M Luck q|ई \\ † Service de Physique de l'État Condensé, CEA Saclay, 91191 Gif-sur-Yvette \\ cedex, France \\ ๆ Service de Physique Théorique \# CEA Saclay, 91191 Gif-sur-Yvette cedex, \\ France
}

\begin{abstract}
Dynamical urn models, such as the Ehrenfest model, have played an important role in the early days of statistical mechanics. Dynamical manyurn models generalize the former models in two respects: the number of urns is macroscopic, and thermal effects are included. These many-urn models are exactly solvable in the mean-field geometry. They allow analytical investigations of the characteristic features of nonequilibrium dynamics referred to as aging, including the scaling of correlation and response functions in the two-time plane and the violation of the fluctuation-dissipation theorem. This review paper contains a general presentation of these models, as well as a more detailed description of two dynamical urn models, the backgammon model and the zeta urn model.
\end{abstract}

\section{Prologue}

Urns containing balls, just as dice or playing cards, are ubiquitous in writings on probability theory, reminding us that this branch of mathematics owes its early developments to practical questions arising in playing games. Dynamical urn models, such as the Ehrenfest model, have played an important role in the elucidation of conceptual problems in statistical mechanics. More recently, dynamical many-urn models have been investigated in the mean-field geometry. These exactly solvable models exhibit characteristic features of nonequilibrium dynamics referred to as aging, such as the scaling of the correlation and response functions in the two-time plane and the violation of the fluctuation-dissipation theorem.

This paper contains a didactic introduction to urn models (Section 1), a presentation of static and dynamical properties of many-urn models (Section 2), a reminder of the main characteristic features of aging (Section 3), and an overview of recent results on two dynamical urn models, namely the backgammon model (Section 4) and the zeta urn model (Section 5).

\section{Urn models}

\subsection{The Ehrenfest urn model}

This model was devised by $\mathrm{P}$ and T Ehrenfest in 1907 [1] in their attempt to critically review Boltzmann's $H$-theorem. It is defined as follows: $N$ balls are distributed in

$\ddagger$ godreche@spec.saclay.cea.fr

$\S$ luck@spht.saclay.cea.fr

\| URA 2306 of CNRS 
two urns (or boxes). At random times, given by a Poisson process with unit rate, a ball is chosen at random, and moved from the box in which it is to the other box.

Let $N_{1}(t)$ (resp. $N_{2}(t)=N-N_{1}(t)$ ) be the number of balls in box number 1 (resp. number 2) at time $t$. For any initial configuration, the system relaxes to equilibrium for infinitely long times. The equilibrium state is characterized by a binomial distribution of the number $N_{1}$ :

$$
f_{k, \text { eq }}=\mathcal{P}_{\text {eq }}\left(N_{1}=k\right)=2^{-N}\left(\begin{array}{l}
N \\
k
\end{array}\right) \quad(k=0, \ldots, N),
$$

reminiscent of the Maxwell-Boltzmann statistics for indistinguishable particles.

As noticed first by Kohlrausch and Schrödinger [2], $N_{1}(t)$ can be viewed as the coordinate of a fictitious walker. The temporal evolution of the occupation probabilities $f_{k}(t)=\mathcal{P}\left(N_{1}(t)=k\right)$ is governed by the master equation

$$
\frac{\mathrm{d} f_{k}(t)}{\mathrm{d} t}=\frac{k+1}{N} f_{k+1}(t)+\frac{N+1-k}{N} f_{k-1}(t)-f_{k}(t) .
$$

Indeed, a move of a ball from box number 1 to box number 2 (resp. from box number 2 to box number 1) yields $k \rightarrow k-1$ (resp. $k \rightarrow k+1$ ), and occurs with a rate $k / N$ (resp. $(N-k) / N)$ per unit time. The master equation (1.2) describes a non-uniformly biased random walk over the integers $0, \ldots, N$ [2, 3]. The first (resp. the second) term in the right-hand side is absent for $k=N$ (resp. $k=0$ ).

The spectrum of relaxation rates of the model can be derived by looking for solutions to (1.2) of the form $f_{k}(t)=\phi_{k} \mathrm{e}^{-\lambda t}$. One thus obtains $\lambda_{m}=2 m / N$, with $m=0, \ldots, N$. The static solution $(m=0)$ is nothing but the equilibrium distribution (1.1). The occupation probabilities have an exponential convergence to their equilibrium values, with a relaxation time

$$
\tau_{\text {eq }}=1 / \lambda_{1}=N / 2 .
$$

There are, however, much larger time scales in the Ehrenfest model. Consider indeed the length of time it takes for box number 1 to get empty, i.e., the first time $t_{0}$ such that $N_{1}\left(t_{0}\right)=0$. This time depends on the initial state and on the whole history of the system. Its mean value $T_{0}=\left\langle t_{0}\right\rangle$ can be evaluated as follows. The equilibrium probability for box number 1 to be empty, $f_{0, \mathrm{eq}}=2^{-N}$, is exponentially small in the number of balls. It is therefore expected that the typical time needed to reach this very rare event scales as

$$
T_{0} \approx 1 / f_{0, \mathrm{eq}}=2^{N} .
$$

This result can be derived in a more rigorous way. Related matters are discussed in References [4, 5]. The process of emptying one of the boxes is therefore characterized by an exponentially large time $T_{0}$. Equation (1.3) can be recast as an Arrheniuslike law:

$$
T_{0} \sim \mathrm{e}^{S_{0}},
$$

where $S_{0}=N \ln 2$ is the entropy difference between the equilibrium state of the model, where each box contains one half of the balls, up to fluctuations, and the configuration where box number 1 is empty. This entropy difference plays the role of a reduced activation energy in (1.4). This is an elementary example of an entropy barrier. 


\subsection{The Monkey urn model}

Let us introduce a variant of the Ehrenfest urn model, which we choose to call the Monkey urn model, because it corresponds to the image of a monkey playing at exchanging balls between two boxes. The key difference with the previous case is that now at each time step a box (either 1 or 2) is chosen at random, and one ball is moved from the chosen box (provided it is non-empty) to the other one. The choice of a box, instead of a ball, induces drastic changes in the statics and dynamics of the model.

The equilibrium state of the Monkey urn model is now characterized by uniform occupation probabilities

$$
f_{k, \text { eq }}=\mathcal{P}_{\text {eq }}\left(N_{1}=k\right)=\frac{1}{N+1} \quad(k=0, \ldots, N) .
$$

The evolution of the occupation probabilities is governed by the master equation

$$
\frac{\mathrm{d} f_{k}(t)}{\mathrm{d} t}=\frac{1}{2}\left(f_{k+1}(t)+f_{k-1}(t)-2 f_{k}(t)\right),
$$

describing a usual random walk over the integers $0, \ldots, N$, with appropriate reflecting boundary conditions.

The relaxation rates of the model read $\lambda_{m}=2 \sin ^{2}(m \pi /(2(N+1)))$, with $m=0, \ldots, N$. The relaxation time reads therefore

$$
\tau_{\text {eq }}=\frac{1}{\lambda_{1}} \approx \frac{2}{\pi^{2}} N^{2},
$$

where the scaling $\tau_{\mathrm{eq}} \sim N^{2}$ originates in the diffusive nature of the motion of balls.

Because of the flatness of the equilibrium distribution (1.5), there are no entropy barriers in the Monkey urn model. In particular, the mean time $T_{0}$ for a box to get empty scales as $N^{2}$, proportionally to the relaxation time (1.6).

\section{Many-urn models: statics and dynamics}

We now turn to the discussion of static and dynamical properties of two classes of many-urn models, generalizing the Ehrenfest and the Monkey urn models described so far [6]. This classification encompasses examples of urn models studied in recent years. The backgammon model [7] is a prototype of the Ehrenfest class, while model B of References [8, 9] and the zeta urn model [9, 6] belong to the Monkey class.

A many-urn model consists of $N$ balls, distributed among $M$ boxes. Box number $i$ contains $N_{i}$ balls, with

$$
\sum_{i=1}^{M} N_{i}=N .
$$

Most investigations of many-urn models have dealt with the thermodynamic limit $(M \rightarrow \infty, N \rightarrow \infty)$, at a fixed density $\rho=N / M$ of balls per box.

\subsection{Statics}

Two ingredients are necessary in order to define static properties of many-urn models within the framework of statistical mechanics: 
A priori statistics. To each configuration $\left\{N_{i}\right\}$ is attributed an a priori statistical weight

$$
W\left(\left\{N_{i}\right\}\right)= \begin{cases}\prod_{i=1}^{M} \frac{1}{N_{i} !} & (\text { Ehrenfest class) } \\ 1 & \text { (Monkey class). }\end{cases}
$$

The formula for the Ehrenfest class generalizes the binomial law (1.1), and corresponds to the Maxwell-Boltzmann prescription for indistinguishable particles. The formula for the Monkey class generalizes the flat distribution (1.5), and formally corresponds to Bose-Einstein statistics for the occupation numbers of quantum states [10].

Hamiltonian. The Hamiltonian of a many-urn model is defined as the sum of independent contributions of boxes, of the form

$$
\mathcal{H}\left(\left\{N_{i}\right\}\right)=\sum_{i=1}^{M} E\left(N_{i}\right),
$$

so that the unnormalized Boltzmann weight of a configuration $\left\{N_{i}\right\}$ at temperature $T=1 / \beta$ is

$$
\mathrm{e}^{-\beta \mathcal{H}\left(\left\{N_{i}\right\}\right)}=\prod_{i=1}^{M} p_{N_{i}},
$$

with

$$
p_{N_{i}}=\mathrm{e}^{-\beta E\left(N_{i}\right)} .
$$

The canonical partition function thus reads

$$
Z(M, N)=\sum_{\left\{N_{i}\right\}} W\left(\left\{N_{i}\right\}\right) \mathrm{e}^{-\beta \mathcal{H}\left(\left\{N_{i}\right\}\right)} \delta\left(\sum_{i} N_{i}, N\right) .
$$

Using (2.1) and (2.3), and a contour-integral representation of the Kronecker symbol, we are left with

$$
Z(M, N)=\oint \frac{\mathrm{d} z}{2 \mathrm{i} \pi z^{N+1}}[P(z)]^{M},
$$

where

$$
P(z)=\sum_{k \geq 0} p_{k} z^{k} \times \begin{cases}1 / k ! & \text { (Ehrenfest class) } \\ 1 & \text { (Monkey class). }\end{cases}
$$

Static properties of many-urn models are therefore entirely encoded in the temperature-dependent generating series $P(z)$ of the Boltzmann weights $p_{k}$ of (2.4). In the thermodynamic limit, at fixed density $\rho$, the free energy per box,

$$
\beta F=-\lim _{M \rightarrow \infty} \frac{1}{M} \ln Z(M, N) \quad(N \approx M \rho),
$$

can be obtained by evaluating the contour integral in (2.5) by the saddle-point method. The saddle-point value $z_{s}$ depends on temperature and density through the equation

$$
\frac{z_{s} P^{\prime}\left(z_{s}\right)}{P\left(z_{s}\right)}=\rho
$$


and the free energy per box reads

$$
\beta F=\rho \ln z_{s}-\ln P\left(z_{s}\right) .
$$

The equilibrium occupation probabilities $f_{k, \text { eq }}$ of box number $i$ do not depend on the box under consideration. (In the sequel we will refer to box number 1 for definiteness.) We have

$$
f_{k, \mathrm{eq}}=\frac{p_{k} z_{s}^{k}}{P\left(z_{s}\right)} \times \begin{cases}1 / k ! & \text { (Ehrenfest class) }, \\ 1 & \text { (Monkey class) }\end{cases}
$$

The above results further simplify at infinite temperature, where $p_{k}=1$, independently of the form of the energy function. Equations (2.5)-(2.9) become

$$
\begin{aligned}
& P(z)=\mathrm{e}^{z}, \quad Z(M, N)=\frac{M^{N}}{N !}, \quad z_{s}=\rho, \\
& \beta F=\rho \ln \rho-\rho, \quad f_{k, \text { eq }}=\mathrm{e}^{-\rho} \frac{\rho^{k}}{k !}
\end{aligned}
$$

for the Ehrenfest class, and

for the Monkey class.

$$
\begin{aligned}
& P(z)=\frac{1}{1-z}, \quad Z(M, N)=\frac{(M+N-1) !}{N !(M-1) !}, \quad z_{s}=\frac{\rho}{\rho+1}, \\
& \beta F=\rho \ln \rho-(\rho+1) \ln (\rho+1), \quad f_{k, \text { eq }}=\frac{\rho^{k}}{(\rho+1)^{k+1}}
\end{aligned}
$$

\subsection{Dynamics}

An elementary step of the dynamics consists in moving a ball from a departure box $d$ to an arrival box $a$. A move is proposed to the system at every time step $\delta t=1 / M$. The precise definition of a dynamical many-urn model should state the following points:

Geometry. The departure box $d$ and the arrival box $a$ have to be connected to each other. Most investigations of dynamical urn models have dealt with the mean-field geometry, where any two boxes are connected to each other.

Dynamical urn models can also be defined on regular lattices in finite dimension, with the convention that urns are located at the sites of the lattice, and that only nearest neighbors are connected.

A priori dynamical rule. The proposed moves are chosen according to some a priori dynamical rule. There are three natural rules [8]:

- Rule A (ball-box): Choose a ball (this defines the departure box $d$ ). Choose an arrival box $a$, connected to $d$.

- Rule B (box-box): Choose a departure box $d$. Choose uniformly a ball in $d$. Choose an arrival box $a$, connected to $d$.

- Rule $C$ (ball-ball): Choose a first ball (this defines the departure box $d$ ). Choose a second ball (this defines the arrival box $a$ ). Propose the move if the boxes $d$ and $a$ are connected.

The urn models described in Section 1 suggest what should be the right relationship between a priori statistics and dynamical rule. It indeed turns out that Rule A corresponds to the Ehrenfest class, while Rule B corresponds to the Monkey class. Rule C corresponds to yet another class of dynamical urn models, with no obvious static counterpart [8]. 
Thermal rule. The following thermal rules respect detailed balance:

- Metropolis: A proposed move is performed with probability

$$
\min (1, \exp (-\beta \Delta \mathcal{H})),
$$

where $\Delta \mathcal{H}=\mathcal{H}\left(\left\{N_{i}^{\prime}\right\}\right)-\mathcal{H}\left(\left\{N_{i}\right\}\right)$ is the energy difference between the configurations $\left\{N_{i}\right\}$ before and $\left\{N_{i}^{\prime}\right\}$ after the proposed move.

- Heat-bath: Among all the possible a priori choices for the arrival box a, one is chosen with an appropriately normalized probability, proportional to the Boltzmann weight $\exp \left(-\beta \mathcal{H}\left(\left\{N_{i}^{\prime}\right\}\right)\right)$ of the configuration after the move.

Throughout the following, we restrict ourselves to the mean-field geometry, and to a homogeneous disordered initial state, such as, e.g., the infinite-temperature equilibrium state (2.10), (2.11).

The key quantities are again the occupation probabilities $f_{k}(t)=\mathcal{P}\left(N_{1}(t)=k\right)$. Their evolution is given by the master equation

$$
\frac{\mathrm{d} f_{k}(t)}{\mathrm{d} t}=\sum_{\ell \geq 0}\left(\pi_{k+1, \ell}(t)+\pi_{\ell, k-1}(t)-\pi_{k, \ell}(t)-\pi_{\ell, k}(t)\right),
$$

where $\pi_{k, \ell}(t)$ denotes the contribution of a move from the departure box $d$, containing $N_{d}=k$ balls, to the arrival box $a$, containing $N_{a}=\ell$ balls.

The a priori dynamical rule yields

$$
\pi_{k, \ell}(t)=f_{k}(t) f_{\ell}(t) W_{k, \ell}(t)\left(1-\delta_{k, 0}\right) \times \begin{cases}k & \text { (Ehrenfest class) }, \\ 1 & \text { (Monkey class). }\end{cases}
$$

The factor $\left(1-\delta_{k, 0}\right)$ accounts for the fact that an actual move only takes place if the departure box $d$ is not empty. The acceptance rates $W_{k, \ell}(t)$ depend on the thermal part of the rule. With the notation (2.4), the rates for the Metropolis rule [9],

$$
W_{k, \ell}=\min \left(1, \frac{p_{k-1} p_{\ell}}{p_{k} p_{\ell+1}}\right),
$$

are independent of time, while those for the heat-bath rule [9],

$$
W_{k, \ell}(t)=\frac{p_{\ell+1}}{p_{\ell}}\left(\sum_{\ell \geq 0} f_{\ell}(t) \frac{p_{\ell+1}}{p_{\ell}}\right)^{-1},
$$

only depend on the label $\ell$ of the arrival box.

In analogy with (1.2), (2.13) can be recast as the master equation of a random walk over the positive integers $(k=0,1, \ldots)$ :

$$
\left\{\begin{array}{rl}
\frac{\mathrm{d} f_{k}(t)}{\mathrm{d} t} & =\mu_{k+1}(t) f_{k+1}(t)+\lambda_{k-1}(t) f_{k-1}(t) \\
& -\left(\mu_{k}(t)+\lambda_{k}(t)\right) f_{k}(t) \\
\frac{\mathrm{d} f_{0}(t)}{\mathrm{d} t} & =\mu_{1}(t) f_{1}(t)-\lambda_{0}(t) f_{0}(t),
\end{array} \quad(k \geq 1),\right.
$$

where

$$
\lambda_{k}(t)=\frac{1}{f_{k}(t)} \sum_{\ell \geq 0} \pi_{\ell, k}(t), \quad \mu_{k}(t)=\frac{1}{f_{k}(t)} \sum_{\ell \geq 0} \pi_{k, \ell}(t)
$$


are, respectively, the hopping rate to the right, corresponding to $a=1$, i.e., $N_{1}=k \rightarrow$ $N_{1}=k+1$, and to the left, corresponding to $d=1$, i.e., $N_{1}=k \rightarrow N_{1}=k-1$. The equation for $f_{0}(t)$ is special, as $\lambda_{-1}(t)=\mu_{0}(t)=0$.

The master equation (2.14) preserves the sum rules

$$
\sum_{k \geq 0} f_{k}(t)=1, \quad \sum_{k \geq 0} k f_{k}(t)=\left\langle N_{1}(t)\right\rangle=\rho,
$$

expressing the conservation of probability and of the number of balls.

The rates $\lambda_{k}(t)$ and $\mu_{k}(t)$, defined in (2.15), depend on the occupation probabilities $f_{k}(t)$ themselves, so that the master equation (2.14) is actually a nonlinear set of first-order equations for the $f_{k}(t)$. The equilibrium occupation probabilities (2.9) are recovered as their unique stationary state.

\section{Equilibrium vs. nonequilibrium dynamics: two-time quantities}

Nonequilibrium properties of dynamical urn models such as the backgammon model $[7$, 8, 11, 12] or the zeta urn model [9, 6] have been extensively studied in recent years.

These models are simple enough to allow analytical investigations, and rich enough to illustrate most features of nonequilibrium dynamics referred to as aging. The latter include the scaling properties of the correlation and response functions of various observables, and of their fluctuation-dissipation ratios. In this section we recall how the temporal evolution of these two-time quantities can be derived within the master-equation formalism of Section 2.

We consider a dynamical urn model of either class, in the thermodynamic limit. For definiteness, our observable will be the local density of balls, defined as the fluctuating population $N_{1}(t)$ of box number 1 , say. The second of the sum rules (2.16) ensures that the mean density $\left\langle N_{1}(t)\right\rangle=\rho$ is constant in time. Its fluctuations $N_{1}(t)-\rho$ exhibits, however, non-trivial dynamical properties, that can be characterized by twotime correlation and response functions, to be defined below. Hereafter, time variables will be such that $0 \leq s \leq t$. The earlier time $s$ is referred to as the waiting time, the later time $t$ as the observation time.

\subsection{Correlation function}

The (local, connected) two-time correlation function of the density is defined as

$$
C(t, s)=\left\langle N_{1}(s) N_{1}(t)\right\rangle-\rho^{2} .
$$

This can be recast as

$$
C(t, s)=\sum_{k \geq 1} k \gamma_{k}(t, s)-\rho^{2},
$$

where the quantities

$$
\gamma_{k}(t, s)=\sum_{j \geq 1} j f_{j}(s) \mathcal{P}\left\{N_{1}(t)=k \mid N_{1}(s)=j\right\}
$$

assume the initial values

$$
\gamma_{k}(s, s)=k f_{k}(s)
$$


at $t=s$. Their temporal evolution for $t \geq s$ is given by a master equation similar to (2.14):

$$
\left\{\begin{array}{rl}
\frac{\partial \gamma_{k}(t, s)}{\partial t} & =\mu_{k+1}(t) \gamma_{k+1}(t, s)+\lambda_{k-1}(t) \gamma_{k-1}(t, s) \\
& -\left(\mu_{k}(t)+\lambda_{k}(t)\right) \gamma_{k}(t, s) \\
\frac{\partial \gamma_{0}(t, s)}{\partial t} & =\mu_{1}(t) \gamma_{1}(t, s)-\lambda_{0}(t) \gamma_{0}(t, s) .
\end{array} \quad(k \geq 1),\right.
$$

The rates $\lambda_{k}(t)$ and $\mu_{k}(t)$, defined in (2.15), only depend on the $f_{k}(t)$, so that (3.1) are linear equations for the $\gamma_{k}(t, s)$.

\subsection{Response function}

The (local) response function measures the influence on the mean density in box number 1 of a perturbation in the canonically conjugate variable, i.e., the local chemical potential acting on the same box.

Suppose that box number 1 is subjected to a small time-dependent chemical potential $\alpha_{1}(t)$, so that the total Hamiltonian of the system is now

$$
\mathcal{H}\left(\left\{N_{i}\right\}\right)=\sum_{i=1}^{M} E\left(N_{i}\right)+\alpha_{1}(t) N_{1} .
$$

The mean density in box number 1 reads

$$
\left\langle N_{1}(t)\right\rangle=\rho+\int_{0}^{t} R(t, s) \alpha_{1}(s) \mathrm{d} s+\cdots,
$$

where only the term linear in $\alpha(s)$ has been written explicitly, and causality has been used. The kernel of the linear response:

$$
R(t, s)=\frac{\delta\left\langle N_{1}(t)\right\rangle}{\delta \alpha_{1}(s)}
$$

is called the two-time response function. The temporal evolution of this function can again be determined by means of the master-equation formalism.

\subsection{Fluctuation-dissipation theorem: equilibrium vs. nonequilibrium behavior}

Dynamical urn models, as any statistical-mechanical model, have a fast convergence to equilibrium, with a finite relaxation time $\tau_{\text {eq }}$, in their high-temperature (disordered) phase. If the earlier time exceeds the relaxation time $\left(s \gg \tau_{\text {eq }}\right)$, the system is at equilibrium. One-time quantities assume their equilibrium values. Two-time quantities, such as the correlation and response functions, are invariant under time translations:

$$
C(t, s)=C_{\mathrm{eq}}(\tau), \quad R(t, s)=R_{\mathrm{eq}}(\tau),
$$

where

$$
\tau=t-s \geq 0
$$

Furthermore, these quantities are related by the fluctuation-dissipation theorem

$$
R_{\mathrm{eq}}(\tau)=-\frac{1}{T} \frac{\mathrm{d} C_{\mathrm{eq}}(\tau)}{\mathrm{d} \tau}
$$

Some many-urn models exhibit a more interesting nonequilibrium behavior at lower temperatures, where the relaxation time $\tau_{\text {eq }}$ becomes either very large or infinite, so that the waiting time $s$ and the observation time $t$ can be much smaller than $\tau_{\text {eq }}$. 
In such a nonequilibrium situation, both time-translation invariance (3.2) and the fluctuation-dissipation theorem (3.3) are violated in general. It has become customary [13, 14, 15, 16] to characterize departure from equilibrium by the fluctuation-dissipation ratio

$$
X(t, s)=T \frac{R(t, s)}{\frac{\partial C(t, s)}{\partial s}} .
$$

In general, this dimensionless quantity depends on both times $s$ and $t$ and on the observable under consideration. It often exhibits a non-trivial scaling behavior in the two-time plane, which is viewed as one of the salient features of aging.

The definition (3.4) can be rephrased in terms of an effective temperature [14]:

$$
R(t, s)=\frac{1}{T_{\mathrm{eff}}(t, s)} \frac{\partial C(t, s)}{\partial s}, \quad T_{\mathrm{eff}}(t, s)=\frac{T}{X(t, s)} .
$$

At equilibrium, the fluctuation-dissipation theorem is valid, so that $X(t, s)=1$ and $T_{\text {eff }}(t, s)=T$. For a nonequilibrium situation started from a disordered initial state, such as (2.10), (2.11), the effective temperature is expected to lie somewhere between the formal initial temperature $T(0)=\infty$ and the final one, $T(\infty)=T$, i.e.,

$$
0 \leq X(t, s) \leq 1 .
$$

These inequalities are indeed obeyed in all the known cases.

\section{The backgammon model}

The backgammon model is a simple example of a system which exhibits slow relaxation due to entropy barriers [7]. This mean-field many-urn model belongs to the Ehrenfest class, with Metropolis dynamics, and the following choice of an energy function:

$$
E\left(N_{i}\right)=-\delta\left(N_{i}, 0\right) \text {. }
$$

The Hamiltonian (2.2) is therefore equal to minus the number of empty boxes, so that balls tend to condensate in fewer and fewer boxes as times passes, at least at low temperature.

\subsection{Statics}

The equilibrium occupation probabilities read

$$
f_{k, \text { eq }}=\mathrm{e}^{-\Lambda_{\mathrm{eq}}} \frac{\Lambda_{\mathrm{eq}}{ }^{k}}{k !} \times \begin{cases}1 & (k \geq 1), \\ \mathrm{e}^{\beta} & (k=0) .\end{cases}
$$

The parameter $\Lambda_{\text {eq }}$, equal to $z_{s}$ in the formalism of Section 2, represents the mean population of a non-empty box. It is related to temperature and density by

$$
\mathrm{e}^{\beta}=1+\left(\frac{\Lambda_{\mathrm{eq}}}{\rho}-1\right) \mathrm{e}^{\Lambda_{\mathrm{eq}}},
$$

implying

$$
\Lambda_{\mathrm{eq}} \approx \beta-\ln \frac{\beta}{\rho}
$$

at low temperature. The roughly linear divergence of $\Lambda_{\text {eq }}$ is the signature of a zerotemperature condensation transition. The density plays no particular role in this phase transition, so that we set $\rho=1$ in the sequel. 
The slow energy relaxation at low temperature can be understood, at least qualitatively, in terms of the concept of entropy barrier, introduced for the Ehrenfest model. The late stages of the dynamics indeed consist in emptying boxes whose typical population is $\Lambda_{\text {eq }}$. The formula (1.3) suggests the scaling law $\ln \tau_{\text {eq }} \sim \Lambda_{\text {eq }} \sim \beta$. The relaxation time of the energy is indeed known [12] to read

$$
\begin{aligned}
\tau_{\mathrm{eq}} & \approx \frac{\Lambda_{\mathrm{eq}}-1}{\Lambda_{\mathrm{eq}}} I\left(\Lambda_{\mathrm{eq}}\right) \approx \frac{\mathrm{e}^{\Lambda_{\mathrm{eq}}}}{\Lambda_{\mathrm{eq}}}\left(1+\frac{1}{\Lambda_{\mathrm{eq}}^{2}}+\cdots\right) \\
& \approx \frac{\mathrm{e}^{\beta}}{\beta^{2}}\left(1+\frac{2 \ln \beta+1}{\beta}+\cdots\right)
\end{aligned}
$$

at low temperature, with the other characteristic times remaining of order $\Lambda_{\text {eq. }}$. The function

$$
I(\Lambda)=\int_{0}^{1} \mathrm{~d} z \frac{\mathrm{e}^{\Lambda z}-1}{z}=\operatorname{Ei}(\Lambda)-\ln \Lambda-\gamma,
$$

where Ei is the exponential integral and $\gamma$ is Euler's constant, plays a central role in the analysis of the low-temperature regime.

\subsection{Nonequilibrium dynamics: logarithmic relaxation}

In the present case, the master equation (2.14) for the occupation probabilities reads

$$
\left\{\begin{array}{l}
\frac{\mathrm{d} f_{k}(t)}{\mathrm{d} t}=\frac{k+1}{\Lambda(t)} f_{k+1}(t)+f_{k-1}(t)-\left(1+\frac{k}{\Lambda(t)}\right) f_{k}(t) \quad(k \geq 2), \\
\frac{\mathrm{d} f_{1}(t)}{\mathrm{d} t}=\frac{2}{\Lambda(t)} f_{2}(t)+\mu(t) f_{0}(t)-2 f_{1}(t), \\
\frac{\mathrm{d} f_{0}(t)}{\mathrm{d} t}=f_{1}(t)-\mu(t) f_{0}(t),
\end{array}\right.
$$

with

$$
\frac{1}{\Lambda(t)}=1-\left(1-\mathrm{e}^{-\beta}\right) f_{0}(t), \quad \mu(t)=\mathrm{e}^{-\beta}+\left(1-\mathrm{e}^{-\beta}\right) f_{1}(t)
$$

The parameter $\Lambda(t)$ again represents the mean population of a non-empty box in the low-temperature regime, while the energy density reads

$$
E(t)=-f_{0}(t) \approx-1+\frac{1}{\Lambda(t)}
$$

The nonequilibrium regime of interest, called the alpha regime in the context of the physics of glasses, corresponds to low temperature and long times. It is natural to investigate the slow relaxation dynamics in this regime by means of an adiabatic decoupling, similar to the Born-Oppenheimer scheme, between the slow evolution of $\Lambda(t)$, due to emptying new boxes, generalizing the time scale $\tau_{\text {eq }}$, and the fast evolution of the other degrees of freedom, corresponding to the diffusive motion of balls between non-empty boxes, generalizing the relaxation times of order $\Lambda_{\text {eq }}$.

This decoupling scheme can be implemented in several ways. An analytical approach, extensively used in [12], is based on the asymptotic analysis of formal integral representations of the solution to the master equation (4.2). It amounts to neglecting only exponentially small terms, either in $\Lambda$ or in $\Lambda_{\text {eq }}$. This approach shows that $\Lambda(t)$ follows an effective Markovian dynamics

$$
\frac{\mathrm{d} \Lambda}{\mathrm{d} t} \approx \frac{1}{I(\Lambda(t))}\left(1-\frac{(\Lambda(t)-1) \mathrm{e}^{\Lambda(t)}}{\left(\Lambda_{\mathrm{eq}}-1\right) \mathrm{e}^{\Lambda_{\mathrm{eq}}}}\right)
$$


throughout the alpha regime $\left(\Lambda(t)\right.$ and $\Lambda_{\text {eq }}$ both large $)$.

At zero temperature, the large parenthesis in (4.3) equals unity, so that

$$
\Lambda(t) \approx \ln t+\ln \ln t+\frac{\ln \ln t-2}{\ln t}+\cdots
$$

The same logarithmic law is present at finite temperature in the aging regime, namely for $\Lambda(t) \ll \Lambda_{\text {eq }}$, i.e., $t \ll \tau_{\text {eq }}$. It is the nonequilibrium counterpart of the equilibrium relationship (4.1) between $\tau_{\text {eq }}$ and $\Lambda_{\text {eq }}$.

\subsection{Nonequilibrium dynamics: two-time quantities}

The two-time quantities defined in Section 3 have been studied for both local energy and density fluctuations, by means of the above adiabatic approach [12]. The main results are as follows.

Throughout the alpha regime, the two-time correlation and response functions exhibit a leading power-law scaling, with prefactors which depend logarithmically on both times $s$ and $t$. Consider for definiteness zero-temperature dynamics. The energy and density correlation functions read

$$
C_{E}(t, s) \approx \frac{\Phi(s)}{\Phi(t)}, \quad C_{D}(t, s) \approx(\Lambda(s)-1) \frac{\Lambda(t) \Phi(s)}{\Lambda(s) \Phi(t)},
$$

with

$$
\begin{aligned}
\Phi(t) & =\exp \left(\int_{1}^{\Lambda(t)} \frac{\mathrm{d} \Lambda}{\Lambda} \frac{\Lambda^{2} \mathrm{e}^{-\Lambda} I(\Lambda)+1}{\Lambda^{2} \mathrm{e}^{-\Lambda} I(\Lambda)+1-\Lambda}\right) \\
& =\sqrt{t} \ln t\left(1+\frac{\ln \ln t-2}{\ln t}+\cdots\right),
\end{aligned}
$$

so that their leading long-time behavior is

$$
C_{E}(t, s) \approx \sqrt{\frac{s \ln s}{t \ln t}}, \quad C_{D}(t, s) \approx \sqrt{\frac{s}{t}} \ln s .
$$

Similar scaling expressions can be obtained for the local energy and density response functions $R_{E}(t, s)$ and $R_{D}^{ \pm}(t, s)$. It turns out that two density response functions have to be considered, as a peculiarity of Metropolis dynamics, because the acceptance rate (2.12) is not differentiable (it has a cusp) at $\Delta \mathcal{H}=0$. The associated fluctuation-dissipation ratios are independent of the observation time $t$ throughout the alpha regime, provided $\tau=t-s \gg 1$ is a macroscopic time.

For a large waiting time $s$, one has

$$
\begin{aligned}
& X_{E}(t, s) \approx 1-\frac{2}{\Lambda(s)^{2}}-\frac{4}{\Lambda(s)^{3}}-\frac{6}{\Lambda(s)^{4}}+\cdots \\
& X_{D}^{+}(t, s) \approx 1-\frac{2}{\Lambda(s)^{2}}-\frac{2}{\Lambda(s)^{3}}-\frac{12}{\Lambda(s)^{4}}+\cdots \\
& X_{D}^{-}(t, s) \approx 1-\frac{3}{\Lambda(s)^{2}}-\frac{3}{\Lambda(s)^{3}}-\frac{15}{\Lambda(s)^{4}}+\cdots
\end{aligned}
$$

The fluctuation-dissipation theorem is thus asymptotically restored in the longtime regime, with a leading logarithmic correction in $1 /(\ln s)^{2}$, which depends both on the observable (energy vs. density fluctuations) and on microscopic details of the model (Metropolis vs. heat-bath dynamics). 


\section{The zeta urn model}

The static zeta urn model has been introduced 117] as a mean-field model of discretized quantum gravity. Its dynamics has been subsequently investigated [9, 6]. The zeta urn model belongs to the Monkey class, with mean-field heat-bath dynamics, defined by the energy function

$$
E\left(N_{i}\right)=\ln \left(N_{i}+1\right) .
$$

\subsection{Statics}

The logarithmic confining potential (5.1) is such that the Boltzmann weight

$$
p_{N_{i}}=\left(N_{i}+1\right)^{-\beta}
$$

falls off as a power law, with a temperature-dependent exponent. The corresponding generating series has a power-law singularity at $z_{c}=1$, of the form $P_{\mathrm{sg}}(z) \sim z^{\beta-1}$, allowing a condensation transition for finite values of temperature and density. At low enough temperature $(\beta>2), P_{\mathrm{sg}}(z)$ vanishes more rapidly than linearly as $z \rightarrow 1$, so that there is a finite critical density, corresponding to $z_{s}=z_{c}=1$, namely

$$
\rho_{c}=\frac{P^{\prime}(1)}{P(1)}=\frac{\zeta(\beta-1)-\zeta(\beta)}{\zeta(\beta)},
$$

where $\zeta$ denotes Riemann's zeta function, which also enters (5.2), hence the name of the model, proposed in [6]. The static phase diagram of the model is as follows [17]:

Fluid phase $\left(\rho<\rho_{c}\right)$. In this low-density phase we have $z_{s}<1$, so that the equilibrium occupation probabilities (2.9) decay exponentially. The density goes continuously to its critical value $\left(\rho \rightarrow \rho_{c}^{-}\right)$as $z_{s} \rightarrow 1^{-}$.

Criticality $\left(\rho=\rho_{c}\right)$. Along the critical line in the temperature-density plane, the equilibrium occupation probabilities

$$
f_{k, \mathrm{eq}}=\frac{(k+1)^{-\beta}}{\zeta(\beta)}
$$

obey a zeta distribution, with a temperature-dependent exponent. More generally, critical exponents and other universal quantities depend continuously on temperature along the critical line. The variance of the population of box number 1 :

$$
C_{\mathrm{eq}}=\sum_{k \geq 0} k^{2} f_{k, \mathrm{eq}}-\rho_{c}^{2}=\frac{\zeta(\beta) \zeta(\beta-2)-\zeta(\beta-1)^{2}}{\zeta(\beta)^{2}},
$$

is finite for $\beta>3$ (regular critical regime), while it is infinite in the strong-fluctuation critical regime $(2<\beta<3)$.

Condensed phase $\left(\rho>\rho_{c}\right)$. A macroscopic condensate appears in this high-density phase. Indeed, the static configurations are such that the critical occupation probabilities (5.2) still hold for all boxes but one, in which an extensive number of balls, of order $N-M \rho_{c}=M\left(\rho-\rho_{c}\right)$, is condensed. 


\subsection{Nonequilibrium critical dynamics $\left(\rho=\rho_{c}\right)$}

Nonequilibrium properties of the zeta urn model have been studied recently [6, both at criticality and in the condensed phase, pursuing earlier investigations [9]. The most salient results are as follows.

Consider the situation of regular criticality $(\beta>3)$, with a disordered initial state such as (2.11). For any large but finite time $t$, the system looks critical, i.e., the occupation probabilities $f_{k}(t)$ have essentially converged toward their equilibrium values (5.2), for $k \ll t^{1 / 2}$, while for $k \gg t^{1 / 2}$ the system still looks disordered, i.e., the $f_{k}(t)$ fall off very fast. At a quantitative level, this crossover behavior is described by the scaling law

$$
f_{k}(t) \approx f_{k, \mathrm{eq}} F(u), \quad u=k t^{-1 / 2} .
$$

The scaling function $F(u)$ is determined by explicitly solving the differential equation describing the continuum limit of the master equation (2.14). Among other consequences, the variance of the population of box number 1 is found to converge to its equilibrium value (5.3) as a power law:

$$
C(t, t)=\left\langle N_{1}(t)^{2}\right\rangle-\rho_{c}^{2} \approx C_{\mathrm{eq}}-\frac{2^{3-\beta} t^{-(\beta-3) / 2}}{(\beta-3) \Gamma((\beta+1) / 2) \zeta(\beta)} .
$$

The two-time local density correlation and response functions have been investigated along the same lines [6]. In the nonequilibrium scaling regime $(s, t \gg 1)$, these two-time quantities are found to scale as

$$
\begin{aligned}
C(t, s) & \approx s^{-(\beta-3) / 2} \Phi(x), \\
\frac{\partial C(t, s)}{\partial s} & \approx s^{-(\beta-1) / 2} \Phi_{1}(x), \\
R(t, s) & \approx s^{-(\beta-1) / 2} \Phi_{2}(x),
\end{aligned}
$$

where the dimensionless time ratio

$$
x=t / s \geq 1
$$

is the temporal analogue of an aspect ratio. As a consequence, the fluctuation-dissipation ratio $X(t, s)$ only depends on $x$ in the scaling regime:

$$
X(t, s) \approx \mathcal{X}(x)=T \frac{\Phi_{2}(x)}{\Phi_{1}(x)} .
$$

The above results illustrate general predictions on nonequilibrium critical dynamics 18, 19, 20, 21]. The exponent of the waiting time $s$ in the first line of (5.6) already appears in (5.5). It is related to the anomalous dimension of the observable under consideration, and would read $(d-2+\eta) / z_{c}$ for a $d$-dimensional ferromagnet, with $\eta$ being the equilibrium correlation exponent and $z_{c}$ the dynamical critical exponent. The scaling functions $\Phi(x), \Phi_{1,2}(x)$ are universal up to an overall multiplicative constant, and they obey a common power-law fall-off in $x^{-\beta / 2}$. The latter exponent is not related to exponents pertaining to usual (equilibrium) critical dynamics. It would read $-\lambda_{c} / z_{c}=\Theta_{c}-d / z_{c}$ for a ferromagnet, where $\lambda_{c}$ is the critical autocorrelation exponent 22 and $\Theta_{c}$ is the critical initial-slip exponent [18].

As a consequence, the dimensionless scaling function $\mathcal{X}(x)$ is universal, and it admits a non-trivial limit value in the regime where both time variables are well separated in the scaling regime:

$$
X_{\infty}=\lim _{s \rightarrow \infty} \lim _{t \rightarrow \infty} X(t, s)=\mathcal{X}(\infty) .
$$


Explicit expressions for the above scaling functions have been derived, using a spectral decomposition in Laguerre polynomials. Let us mention that the limit fluctuation-dissipation ratio,

$$
X_{\infty}=\frac{\beta+1}{\beta+2} \quad(\beta>3),
$$

lies in an unusually high range $\left(4 / 5<X_{\infty}<1\right)$ for a critical system. Indeed, statistical-mechanical models such as ferromagnets are observed to have $0<X_{\infty} \leq 1 / 2$ at their critical point. The upper bound $X_{\infty}=1 / 2$, corresponding to the mean-field situation [20], is also observed in a range of simpler models [13, 15, 16, 19].

\subsection{Nonequilibrium condensation dynamics $\left(\rho>\rho_{c}\right)$}

Consider now a disordered initial condition such as (2.11) with $\rho>\rho_{c}$, so that the system will build up a macroscopic condensate. The main results concerning this situation are as follows [6].

The growth of the condensate exhibits a scaling regime for $1 \ll t \ll M^{2}$, while finite-size effects become dominant for later times. In this scaling regime, the occupation probabilities $f_{k}(t)$ have essentially converged toward their equilibrium values (5.2) for $k \ll t^{1 / 2}$, while each box has a small probability, of order $t^{-1 / 2}$, to be part of the growing condensate, and to contain a large number of balls, of order $t^{1 / 2}$. This picture can again be turned to a scaling law, which is of the form

$$
f_{k}(t) \approx \frac{F(u)}{t}, \quad u=k t^{-1 / 2} .
$$

Contrary to the scaling function $F(u)$ of (5.4), neither the present scaling function nor those entering (5.7) can be expressed in closed form, because the continuum limit of the master equation (2.14) in the condensed phase involves a biconfluent Heun differential equation, whose solutions are not known explicitly.

The investigation of the scaling regime of condensation dynamics can be extended to two-time quantities $\sqrt{6]}$, which are found to scale as

$$
\begin{aligned}
C(t, s) & \approx\left(\rho-\rho_{c}\right) s^{1 / 2} \Phi(x), \\
\frac{\partial C(t, s)}{\partial s} & \approx\left(\rho-\rho_{c}\right) s^{-1 / 2} \Phi_{1}(x), \\
R(t, s) & \approx\left(\rho-\rho_{c}\right) s^{-1 / 2} \Phi_{2}(x), \\
X(t, s) & \approx \mathcal{X}(x)=T \frac{\Phi_{2}(x)}{\Phi_{1}(x)} .
\end{aligned}
$$

This condensation dynamics exhibits many peculiarities with respect to usual phase-ordering dynamics [23], which takes place, e.g., if a ferromagnet is quenched below its critical temperature. In the latter case, domain growth and phase separation take place in a statistically homogeneous way, at least for an infinite system. To the contrary, in the present situation, condensation takes place in a very inhomogeneous fashion, as fewer and fewer boxes are involved.

Among unusual features of two-time quantities, let us emphasize that the scaling functions $\Phi(x), \Phi_{1,2}(x)$ assume finite values, both at coinciding times $(x=1)$ and in the limit of large time separations $(x=\infty)$. Furthermore, the limit fluctuationdissipation ratio $X_{\infty}=\mathcal{X}(\infty)$ depends continuously on temperature throughout the condensed phase $(\beta>2$, i.e., $T<1 / 2)$, and it vanishes only as

$$
X_{\infty}=T^{1 / 2}-\frac{T^{3 / 4}}{4}+\cdots
$$


at low temperature, while coarsening systems are known [24 to have identically $X_{\infty}=0$ throughout their low-temperature phase.

\section{References}

[1] Ehrenfest P and T 1907 Phys. Zeit. 8311

[2] Kohlrausch F and Schrödinger E 1926 Phys. Zeit. 27306

[3] Kac M 1947 Amer. Math. Monthly 54369

Kac M 1959 Probability and Related Topics in Physical Sciences Lectures in Applied Mathematics vol 1 A (American Mathematical Society)

Siegert A J F 1949 Phys. Rev. 761708

Hess F G 1954 Amer. Math. Monthly 61323

[4] Lipowski A 1997 J. Phys. A: Math. Gen. 30 L91

[5] Murthy K P N and Kehr K W 1997 J.Phys. A: Math. Gen. 306671

[6] Godrèche C and Luck J M 2001 cond-mat/0106272

[7] Ritort F 1995 Phys. Rev. Lett. 751190

[8] Godrèche C, Bouchaud J P, and Mézard M 1995 J. Phys. A: Math. Gen. 28 L603

[9] Drouffe J M, Godrèche C, and Camia F 1998 J. Phys. A: Math. Gen. 31 L19

[10] Kim B J, Jeon G S, and Choi M Y 1996 Phys. Rev. Lett. 764648

[11] Franz S and Ritort F 1995 Europhys. Lett. 31507

Franz S and Ritort F 1996 J. Stat. Phys. 85131

Franz S and Ritort F 1997 J. Phys. A: Math. Gen. 30 L359

[12] Godrèche C and Luck J M 1996 J. Phys. A: Math. Gen. 291915

Godrèche C and Luck J M 1997 J. Phys. A: Math. Gen. 306245

Godrèche C and Luck J M 1999 J. Phys. A: Math. Gen. 326033

[13] Cugliandolo L F and Kurchan J 1994 J. Phys. A: Math. Gen. 275749

Cugliandolo L F, Kurchan J, and Parisi G 1994 J. Phys. I (France) 41641

[14] Cugliandolo L F, Kurchan J, and Peliti L 1997 Phys. Rev. E 553898

[15] Vincent E, Hammann J, Ocio M, Bouchaud J P, and CugliandoloL L 1997 Complex Behavior of Glassy Systems Springer Lecture Notes in Physics 492 184, cond-mat/9607224

[16] Bouchaud J P, Cugliandolo L F, Kurchan J, and Mézard M 1998 Spin Glasses and Random Fields Directions in Condensed Matter Physics vol 12 Young A P ed (Singapore: World Scientific), cond-mat/9702070

[17] Bialas P, Burda Z, and Johnston D 1997 Nucl. Phys. B 493505

Bialas P, Burda Z, and Johnston D 1999 Nucl. Phys. B 542413

Bialas P, Bogacz L, Burda Z, and Johnston D 2000 Nucl. Phys. B 575599

[18] Janssen H K, Schaub B, and Schmittmann B 1989 Z. Phys. B 73539

[19] Godrèche C and Luck J M 2000 J. Phys. A: Math. Gen. 331151

[20] Godrèche C and Luck J M 2000 J. Phys. A: Math. Gen. 339141

[21] Godrèche C and Luck J M 2001 Nonequilibrium critical dynamics of ferromagnetic spin systems in this volume

[22] Huse D A 1989 Phys. Rev. B 40304

[23] Bray A J 1994 Adv. Phys. 43357

[24] Cugliandolo L F, Kurchan J, and Peliti L 1997 Phys. Rev. E 553898

Barrat A 1998 Phys. Rev. E 573629

Berthier L, Barrat J L, and Kurchan J 1999 Eur. Phys. J. B 11635 